\title{
VISIBLE LIGHT COMMUNICATIONS IN HETEROGENEOUS NETWORKS: PAVING THE WAY FOR USER-CENTRIC DESIGN
}

\author{
Rong Zhang, Jiaheng Wang, Zhaocheng Wang, Zhengyuan Xu, Chunming Zhao, \\ AND LAJOS HANZO
}

Rong Zhang and Lajos

Hanzo are with the

University of

Southampton.

Jiaheng Wang and

Chunming Zhao are with

Southeast University.

Zhaocheng Wang is with Tsinghua University.

Zhengyuan Xu is with USTC.

The financial support of the EPSRC under the India-UK Advanced Technology Centre (IUATC), that of the EU under the Concerto project as well as that of the European Research Council's (ERC) Advanced Fellow Grant is gratefully acknowledged. This work is also supported in part by the National Key Basic Research Program of China (Grant No. 2013CB329201).

\begin{abstract}
At the time of this writing, there is substantial research interest in the subject of visible light communications (VLC) owing to its ability to offer significant traffic offloading potential in highly crowded radio frequency (RF) scenarios. We introduce the user-centric design of VLC for heterogeneous networks (HetNet), where three key aspects are identified and elaborated on: signal coverage quality, system control, and service provision aspects. More explicitly, the concepts of amorphous cell formation as well as separated up-link (UL) and down-link (DL), decoupled data and control, dynamic load balancing (LB), etc., all demand radically new thinking. The advocated user-centric VLC design is of key significance in the small-cell scenarios of the emerging $5 \mathrm{G}$ design philosophy.
\end{abstract}

\section{INTRODUCTION}

\section{BACKGROUND AND MOTIVATION}

At the time of this writing, visible light communications (VLC) constitutes a rapidly developing research area, drawing significant attention both from academia and industry. Historically, the first experiment of VLC can be traced back to the old English beacon system relying on bonfires lit on top of regularly-spaced beacon-hills. Then in the late 19th-century, Alexander Graham Bell invented the photophone by transmitting speech over modulated sunlight. This pioneering experiment inspired the recent implementation of data transmission using light emitting diodes (LEDs) by the Nakagawa laboratory [1] in 2004 and raised a significant amount of investments, as exemplified by the EU-FP7 project OMEGA (http://www.ict-omega.eu/). Moreover, several collaborative projects were launched across the globe, such as the VLC standardization process (http://www.ieee802.org/15/pub/ TG7.html), the LiFi Consortium (http://www.lificonsortium.org/), the VLC Consortium (http:// www.vlcc.net/), etc. Following from these advances, link-level data rates attaining hundreds of Mega-bits/sec have been reported using state-of-the-art LEDs and photo-detectors [2], while a variety of low-rate indoor positioning applications have also been commercialized (http://www.bytelight.com/).

The development of VLC solutions essentially relies on the maturing wireless communications techniques and on the gradually improving performance as well as on the growing popularity of LEDs. It is envisioned that LEDs will dominate the general illumination market owing to their energy-efficiency, color-rendering capability and longevity. Thus, LEDs constitute pervasive luminaries providing an attractive modulation potential in relation to the current technology. By modulating data on the visible light produced by the LEDs way above the human eye's fusion rate, the dual goal of communication and illumination can be realized simultaneously. To elaborate a little further, in their most basic form, VLC systems generally exploit intensity modulation at the LED transmitters, which can be readily detected by the photo-diode (PD) receivers. The attainable link-level data rates can be improved from using simple on-off keying (OOK) and pulse position modulation (PPM), to employing more sophisticated modulation schemes, such as the Optical Orthogonal Frequency Division Modulation (OOFDM) [3, 4]. More ambitiously, Giga-bits/sec transmissions have also been achieved with the aid of multiple input multiple output (MIMO) techniques [5]. Even higher link-level data rates are achievable by using the RGB-LED with Wavelength Division Multiplexing (WDM) [6].

The advantages of VLC are multi-fold, including using the unlicensed spectrum, availability of vast bandwidths, the presence of a ubiquitous lightening infrastructure, energy efficiency, etc. Naturally, disadvantages also exist, such as a confined coverage, sensitivity to lineof-sight (LoS) blocking as well as sun-light, and the lack of up-link (UL) support, etc. Nonetheless, there have been tremendous efforts invested in increasing the link-level data-rate of VLC 
by improving the LED components and the physical-layer transmission techniques of VLC, such as the recent research of ultra-parallel VLC relying on gallium nitride ( $\mathrm{GaN})$ opto-electronics (http://up-vlc.photonics.ac.uk/). On commercialization, a recent report [7] suggests that the indoor VLC market is expected to grow from around $\$ 83$ million in 2012 to approximately $\$ 4.5$ billion in 2018, increasing at a rate of approximately 84 percent annually. To facilitate this, two different industries, namely the lighting and mobile sectors, have to work together in order to bring VLC to the market. Finally, for further information on standardization and commercialization we refer readers to [8].

\section{SCOPE AND ORGANISATION}

In order to fully exploit the benefits of VLC, the system-level study of VLC must be further developed to broaden its scope beyond point-to-point applications [9]. From a networking perspective, VLC can be considered as a new member in the small-cell family of the heterogeneous networks (HetNet) landscape for complementing the overloaded radio frequency ( $\mathrm{RF}$ ) communications [10-13]. Naturally, some challenges arise when incorporating VLC into HetNet environments, which may require a radical paradigm shift and new system architectures. This leads to the proposed user-centric design, which can be deemed to be one of the disruptive techniques in the forthcoming $5 \mathrm{G}$ era [14].

The axiomatic role of "cells" has been fundamental to wireless networks. Traditionally, the nature of a cell-centric design is that cell planning, resource management, mobility control, service provision, signal processing, etc., are all unilaterally carried out by the base station (BS). Within the new paradigm of HetNets, consisting of multi-tier and super-dense cell combinations, the current architecture and design principle fails to provide native support for them. Hence, novel concepts such as liquid cells, soft cells, phantom cells, and cloud radio access networks (RAN) have been proposed as potential radio access architectures [15]. Moreover, the emerging device-to-device (D2D) as well as smart content caching and mobile social networks also call for an architectural evolution, where the center of gravity moves from the network side to the smart periphery. These innovative ideas and new user scenarios all led to the radical concept of user-centric design, which is a recent architectural trend for constructing $5 \mathrm{G}$ wireless networks, where the empowered end user will actively participate as a network element, instead of being a simple endpoint. User-centric design exploits the end users' spontaneous activities in geo-location and their diverse service requirements, which is facilitated by the fact that smart phones have become so powerful and mobile cloud computing is also emerging. To elaborate a little further, important concepts in user-centric design include amorphous cells, decoupled signalling and control, as well as separated down-Link (DL) and UL provision. All of these will be treated in this overview paper.

More explicitly, the user-centric VLC HetNet design is highlighted from three perspectives as follows:
- Signal coverage: We redefine the concept of VLC cells, where the underlying dilemma is the choice between fixed-shape confined cells according to the conventional cellular system architecture, and irregular-shaped elastic cells from a user-centric design perspective.

- System control: We reformulate the relationship between data and control by exploiting the naturally separated DL and UL transmissions, where we assign the control function to an umbrella radio access technology (RAT) over-sailing above the VLC cells. ${ }^{1}$

- Service provision: We improve the quality of service (QoS) from a user-centric perspective by treating the VLC and RAT solutions as collaborative data pipes, which rely on dynamic traffic offloading and multi-homing for seamlessly conveying the tele-traffic.

Our paper is organized as follows. We discuss both the traditional and the user-centric VLC cell formation. This is followed by our user-centric system control and service provision solutions. We list a range of open challenges and conclude the article. Finally, the parameters used for our simulations in this paper are:

1 Transmitted optical power per VLC AP: 20 W (constituting $20 \times 20$ LED array with a transmitted optical power per LED: $50 \mathrm{~mW}$ ).

2 Semi-angle of half power: $60^{\circ}$.

3 Physical area of PD: $1 \mathrm{~cm}^{2}$.

4 Gain of optical filter: 1.

5 Refractive index: 1.5.

6 O/E conversion efficiency: $0.53 \mathrm{~A} / \mathrm{W}$.

7 Modulation scheme: PAM.

8 Available bandwidth: $20 \mathrm{MHz}$.

9 Height of VLC AP: $2.5 \mathrm{~m}$.

10 Height of user: $0.85 \mathrm{~m}$.

Remark: Our exploitation of user-centric design is based on PD type receivers, but the proposed concepts are generally also applicable to other receivers. Specifically, image sensor based receivers are promising and hence worth pursuing in our future work.

\section{BREAKING BOUNDARIES}

Let us now focus our attention on the user-centric signal coverage in indoor VLC environments, albeit they are equally applicable in outdoor environments. When multiple $L E D$ arrays are installed, which are referred to as VLC access points (APs), the coverage area of a particular AP is defined as the area receiving the combined LoS ray and the first reflected ray, provided that its power is higher than a certain minimum acceptable value. To elaborate a little further, the minimum acceptable value determines the coverage, below which no service could be supported. Hence this concept is reminiscent of the received signal strength indicator (RSSI) defined in mobile networks. The contributions from farther reflections are excluded, since they tend to be small. ${ }^{2}$ The propagation of visible light is characterized by a confined coverage, which depends mainly on both the transmitter's semi-angle of half power and the receiver's field of view (FoV). Let us discuss three different VLC cell formations, ranging from the traditional cellular perspective to a radical usercentric design.
1 There are many types of RATs that can be considered as umbrella networks for VLC. The typical examples include femto-cells and WiFi for indoor applications as discussed in this paper and pico-cells for outdoor. Moreover, both blue-tooth and infra-red systems may be used for low-rate applications. Generally, multi-tier HetNets can be formed to include micro/macrocells and indoor/outdoor cells, which are also desired to be seamlessly amalgamated.

${ }^{2}$ For a $3 \mathrm{~m} \times 15 \mathrm{~m} \times 15 \mathrm{~m}$ indoor environment having $4 \times 4$ uniformly distributed VLC APs mounted at a height of $2.5 \mathrm{~m}$, at the receiving surface of $0.85 \mathrm{~m}$, the average optical power received from the first reflection accounts for around 3.5 percent of the average optical power received from via the LoS path. Hence, it is reasonable to discard the contributions beyond the second reflections. 


\section{FiXed-SHAPE CELL}

Regular Design: The most straightforward way of constructing a VLC cell is to simply consider each AP function as an individual cell and to adopt the unity frequency reuse across all cells. An example can be seen at the top left corner of Fig. 1a, where the typical problem of inter-cell interference (ICI) imposed by the LoS ray of neighboring cells can be observed at the cell edge. The ICI can be alleviated by the sufficiently confined cell coverage of APs, which provides an inherent degree of spatial reuse to allow for unity frequency reuse, provided that the FoV is narrow. However, this would lead to isolated "coverage islands" and "coverage holes," which consequently result in potentially frequent horizontal handover and outage events when moving between APs, because in the area without LoS coverage, the users will experience dramatic performance degradation, even within a few meters.

Frequency Reuse: The cost of eliminating the "coverage holes" by widening the "coverage islands"

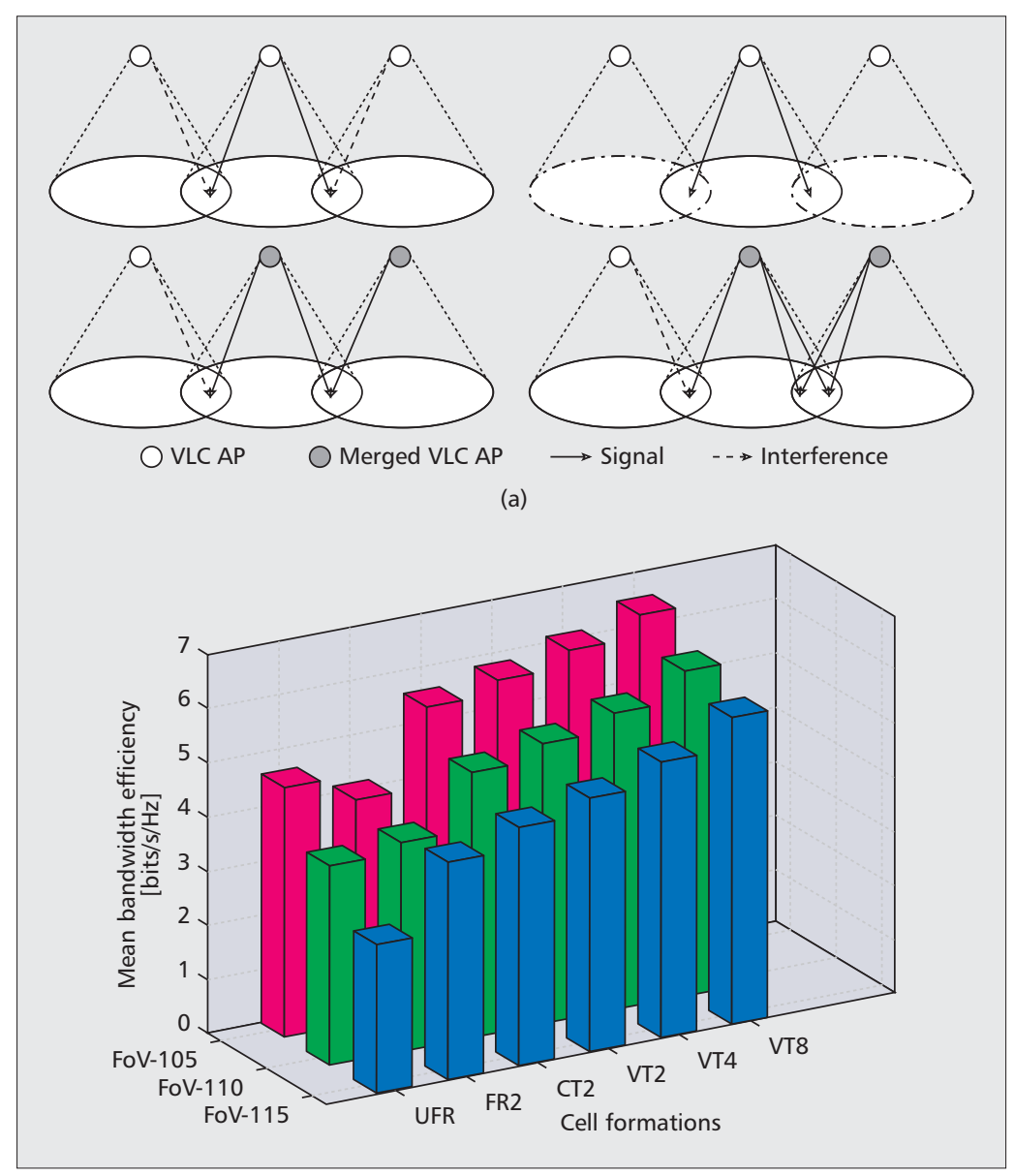

Figure 1. CMean bandwidth efficiency of different fixed-shape cell and merged cell formations as a function of the FoV in a 30-meter line of 8 VLC APs, where "CTx/VTx" denotes the merging of a cluster of "x" cells. Furthermore, UFR and FR2 stand for unity frequency reuse and FRF $=2$ of regular design, respectively. Finally, we also have three FoVs cases, $105^{\circ}$, $110^{\circ}$ and $115^{\circ}$ : a) Illustration of different fixed-shape cell and merged cell formations. The top left is a regular cell formation, the top right has a FRF $=2$, the bottom left represents a merged cell with CT of the same information and the bottom right is a merged cell with VT; and b) mean bandwidth efficiency of different fixed-shape cell and merged cell formations. of APs will increase the cell-edge ICI. Following the traditional cellular design principle, different frequency reuse patterns could be used. Since the first-tier neighboring cells contribute most of the ICI, while the second-tier cells generally have a negligible influence, a frequency reuse factor (FRF) of two is sufficient for providing an acceptable signal to interference plus noise ratio (SINR) at the cell edge, unless the receiver's FOV is extremely wide or there is a large cell overlap. An example can be seen at the top right corner of Fig. 1a, where the solid cell and dashed cell rely on orthogonal frequencies with a $\mathrm{FRF}=2$. Hence, no ICI is observed. Although this is an appealingly simple solution, when using a FRF larger than one, the system has to obey the classic trade-off between reduced bandwidth efficiency and improved SINR. We refer readers to [12] for more technical information. Furthermore, an inherent disadvantage of FR is that switching between VLC frequencies every few meters degrades the user experience.

\section{Merged CelL}

Combined Transmission: An effective method of improving the mobility, while mitigating the detrimental ICI, is to employ cell merging, where a group of neighboring APs jointly form an enlarged VLC cluster. In this way, the coverage of the cell-edge that originally suffered from high ICI is improved by the newly formed merged cell via combined transmission (CT) of multiple APs [12]. In CT, several APs will form a cluster and transmit the same signal to the user. At the user-side, those signals will be added constructively, since optical channels are characterized by real and positive values. Furthermore, if those APs transmitted different signals, then interference would occur. Hence, the potential interference is beneficially turned into useful signals. An example can be seen at the bottom left corner of Fig. 1a, where two APs form a VLC cluster and the previously ICI-contaminated area becomes the cluster-center of CT. As a result, soft horizontal handovers may be triggered across the cells within the cluster. However, a disadvantage of CT is that in the area originally contaminated by ICI, only a single user is served at a time by more than one AP, which are hence potentially under-utilized.

Vector Transmission - In the originally ICI-contaminated area, instead of serving a single user at a time with the aid of CT, multiple users can be served simultaneously by employing proper transmit pre-coding (TPC) techniques, leading to sophisticated vector transmission (VT) [12]. For example, zero-forcing (ZF) based TPC is a well known technique in the multi-user MIMO literature of RF communications. The underlying principle is to totally eliminate the ICI at the multiple transmitters, so that the multiple users receive mutually interference-free signals. An example can be seen at the bottom right corner of Fig. 1a, where two APs form a VT-aided cluster and two users are served simultaneously in the cluster-center with the aid of TPC. In general, to facilitate VT from $N$ APs to $K$ users, both the $(N \times K)$ channel state information $(\mathrm{CSI})$ and the users' data have to be shared among the $N$ 

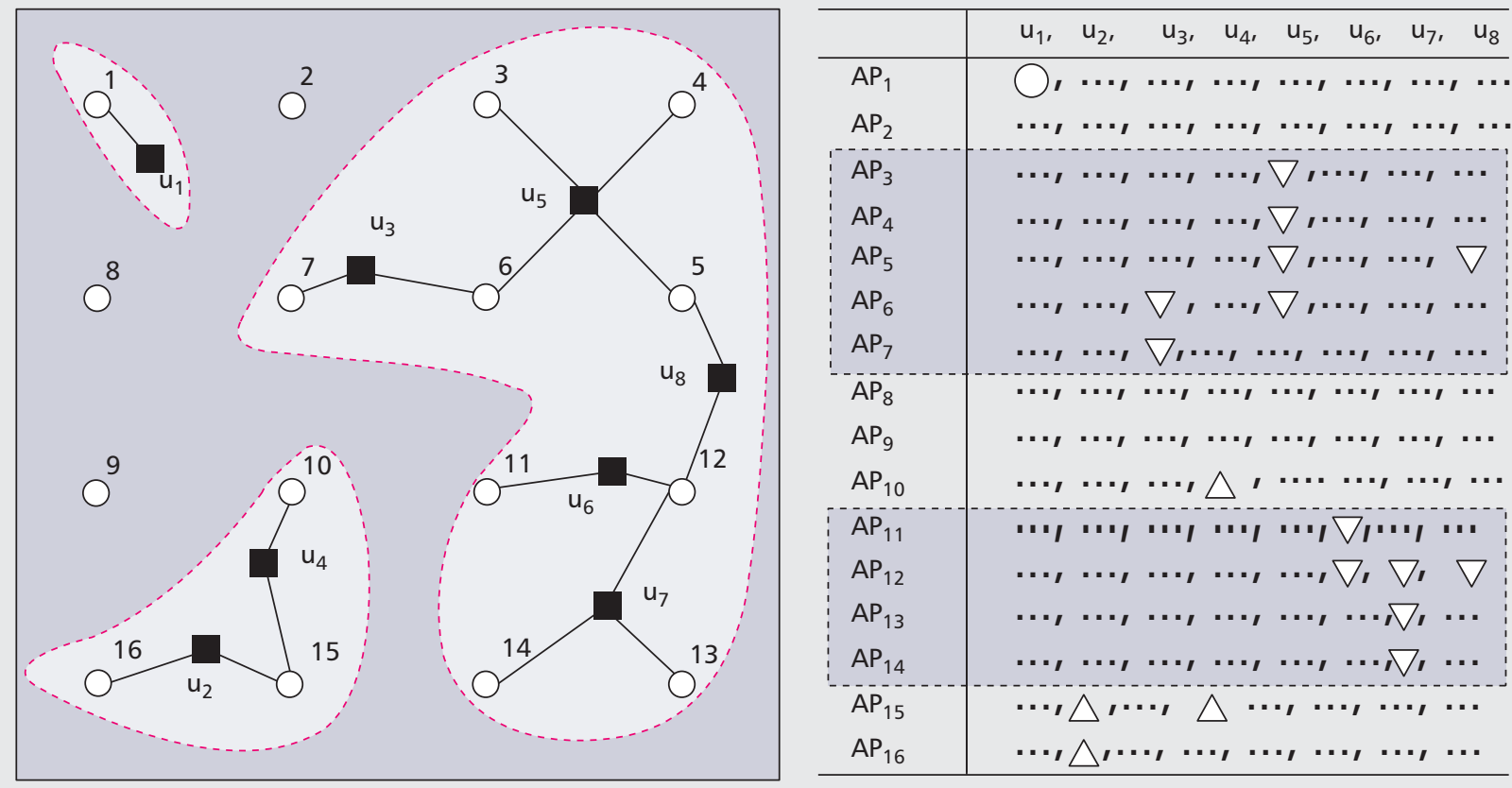

Figure 2. Amorphous cell formation with the table of user-specific AP combinations.

APs. For VLC channels, the CSI may simply be assumed to be an attenuation factor. Fortunately, this requirement can be readily satisfied, since the VLC channels are pre-predominately stationary, which is similar to the successful employment of ZF TPC (known as vectoring) in the state-of-the-art Digital Subscriber Line (DSL) based G.fast system (Recommendation ITU-T G.9701) invoked for coping with the far end cross talk (FEXT) between twisted pairs. Note that VT is reminiscent of the joint transmission concept of the coordinated multiple point (CoMP) transmission regime of RF communications, where we referred to it as VT to highlight its similarity to DSL usage.

Before introducing the user-centric cell formation concept, in Fig. 1b we compared the mean bandwidth efficiency (MBE) of the abovementioned fixed-shape cell and merged cell formations at different FoV in a 30-meter line of eight VLC APs, where "CTx/VTx" represents the merging of a cluster of " $x$ " cells. It is clear that the merged cell formations exhibit the highest MBE, with VT being better than CT, as expected. Moreover, along with the widening of FoV from $105^{\circ}$ to $115^{\circ}$, an increased ICI-contaminated area is observed, leading to the reduction of the MBE for both the separate and for the merged cell formations, while the MBE of employing frequency reuse remains unaffected. Note that when the FoV is $105^{\circ}$, the MBE of FR2 is even lower than that of the regular design, indicating that frequency reuse is a lowcomplexity solution only when severe ICI is encountered.

\section{Amorphous CELLS}

Vector Transmission for Amorhous Cells: Figure 1b suggests that as expected, the VT regime attains its highest MBE when all APs are included in the merged cell, forming a large-scale multi-user
MIMO system. In this case, the entire indoor environment constitutes a single cluster, so that soft horizontal handovers can be applied. However, the associated complexity might become unaffordable. A close inspection reveals that in this large-scale multi-user MIMO system, different users are in fact served by different-sized subsets of the APs, which may or may not be disjoint, as seen at the left of Fig. 2. Hence, this necessitates a user-specific AP combination, as seen in the right of Fig. 2, where for example, $u_{1}$ is only associated with $A P_{1}$ (denoted by a circle) and $u_{2}$ is associated with $A P_{15}, A P_{16}$ (denoted by a triangle), etc. In other words, rather than blindly relating all APs to all users as in the conventional VT, the user-centric design determines which specific subset of APs should serve which particular user. The VT regime is operated on a 'just' sufficient cooperation basis at a reduced complexity. For example, when referring to the table of user-specific AP combinations seen at the right of Fig. 2, the union of two subsets of APs included within the dashed boundary jointly constitute the multi-user MIMO system with respect to the subset of users $\left\{u_{3}, u_{5}, u_{6}, u_{7}, u_{8}\right\}$. Similarly, $\left\{A P_{10}, A P_{15}, A P_{16}\right\}$ simultaneously serve $\left\{u_{2}\right.$, $\left.u_{4}\right\}$ using VT and $A P_{1}$ serves $u_{1}$. Consequently, in our user-centric design, amorphous cells are constructed without a clear cell boundary, since the user-specific AP combinations are of different cardinality. Furthermore, they are updated when the users move, join, or leave the system. This leads to flexible, "breathing" and evolving cells.

Combined Transmission for Amorphous Cells: As described above, VT relies on advanced signal processing techniques and it also requires the CSI information of users to be shared among the APs. If the complexity of VT was unaffordable, a potential simplification is to invoke interference avoidance for the amorphous cells. Let us 


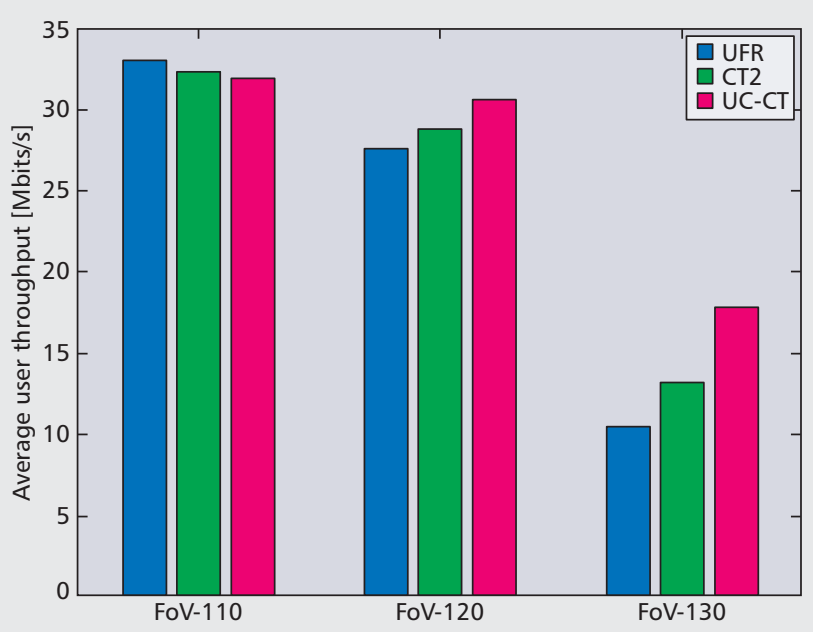

(a)
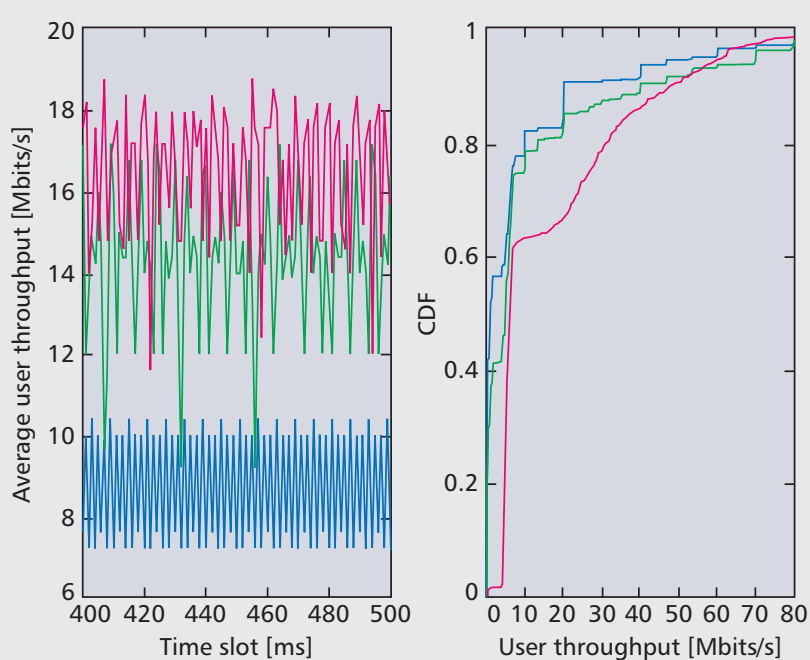

(b)

Figure 3. Simulation results recorded for different cell formations in a $3 \mathrm{~m} \times 15 \mathrm{~m} \times 15 \mathrm{~m}$ indoor environment having $4 \times 4$ uniformly distributed VLC APs, namely the UFR based regular design, the CT of merging two cells into one cluster and the UserCentric CT (UC-CT) for amorphous cells: a) average user throughput of three different cell formations as a function of different FoVs; and b) the left subplot records the snapshot of the average user throughput of three different cell formations between the time slots spanning from $400 \mathrm{~ms}$ to $500 \mathrm{~ms}$ under FoV of $130^{\circ}$, and the right subplot illustrates the Cumulative Distribution Function $(\mathrm{CDF})$ of user throughput of three different cell formations.

assume that a group of $K$ users' user-specific AP combinations have non-empty intersections. Then instead of employing VT for simultaneously serving $K$ users, only a single user can be granted access, which would benefit from CT at any instant, when employing for example proportional-fairness based user-scheduling, so that no interference is generated among the $K$ users. For example, still referring to the table of userspecific AP combinations seen at the right of Fig. 2, the union of two subsets of APs included within the dashed boundary jointly serves one of the users from the set $\left\{u_{3}, u_{5}, u_{6}, u_{7}, u_{8}\right\}$. Mathematically speaking, by forming an interference graph of the user-specific AP combinations, the problem of selecting APs to form amorphous cells can be transformed to the plausible maximum weighted independent set problem that can be efficiently solved by the Greedy Weighted MINimum (GWMIN) algorithm [16]. For other disjoint groups, user-scheduling should be carried out in parallel according to the same algorithm, since there is no interference among the users. Finally, we refer readers to [17] for more technical information.

Figure 3 illustrates our simulation results recorded for different cell formations in a $3 \mathrm{~m} \times$ $15 \mathrm{~m} \times 15 \mathrm{~m}$ indoor environment having $4 \times 4$ uniformly distributed VLC APs, namely the UFR based regular design, the CT of merging two cells into one cluster and the user-centric CT (UC-CT) for amorphous cells. In our simulations, 50 users are randomly placed in the room with a mixture of static and moving users. The edge-effects are eliminated with the aid of the classic wrap-around technique. The moving users follow random directions at a speed of $2 \mathrm{~m} / \mathrm{s}$, where a 30 s period was recorded and proportional-fairness based user-scheduling was employed in conjunction with a slot duration of $1 \mathrm{~ms}$. Figure 3 a shows the average user throughput of three different FoVs. As expected, the overall throughput decreases when the FoV is increased for all three cell formations, and the UC-CT advocated achieves generally a similar or even higher throughput. To gain further detailed insights, the left subplot of Fig. 3 b records the snapshot of the average user throughput between the time slots spanning from $400 \mathrm{~ms}$ to $500 \mathrm{~ms}$ under FoV of $130^{\circ}$, where we can clearly see the superiority of UC-CT. Most importantly, the improved average user throughput of UC-CT does not result in sacrificing the user fairness at all, as illustrated at the right subplot of Fig. 3 b. Indeed, UC-CT also achieves the best user fairness, since all users are served adequately, while more than 40 percent of the users are not served by the rest of the two-cell formations.

\section{EXPLOITING HETEROGENEITY}

The previous section discussed the issues of user-centric VLC cell formation from an ICI perspective. Let us now elaborate further on the user-centric design of VLC in the holistic HetNet environment of Fig. 4.

\section{SEPARATED UL AND DL}

Despite the promise of VLC applications, they may not be operated in isolation, because although LEDs can be used for DL transmission, it is less feasible to embed UL LED transmitters with sufficiently high power into the mobile devices. Consequently, VLC systems may rely on other access techniques for UL transmission. For example, Bluetooth or infra-red are capable of providing low rate UL services, but standard Wireless Fidelity (WiFi) can be more suitable for the UL of indoor environments [10]. Similarly, carrier-grade indoor femto-cells [11] (or outdoor pico-cells) can also be considered as the UL candidate. 
Indeed, supporting the UL and DL through a separate access system is feasible in the usercentric design shown in Fig. 4, where no UL VLC transmissions are used. In addition to the beneficial asymmetric UL and DL rate-assignment capability, an imminent benefit of this flexibility is its full-duplex operation. Indeed, the UL transmission and DL reception of the mobile device can be carried out simultaneously without any self interference (SI), because the RF band and the VLC band rely on completely different physical transceivers. By contrast, in conventional RF systems, the UL and DL APs have to rely on half duplex operation, such as Time Division Duplexing (TDD) or Frequency Division Duplexing (FDD), although the challenges of full-duplex RF operation are also under investigation in the research community.

\section{DeCOUPLED DATA AND CONTROL}

The coexistence of multiple RATs can be exploited for creating a complementary UL service for VLC, whil additionally providing a fall-back solution when the VLC users experience an outage or roam out of range. This will trigger a vertical handover from VLC to the umbrella RAT, which can be operated under the IEEE 802.21 standard, hence supporting seamless media independent handovers (MIH) between networks of different types. In this situation, from a user-centric point of view, a better solution is to decouple data and control signalling, as shown in Fig. 4. More explicitly, the DL data can be conveyed by VLC, while the control signalling can be provided by the umbrella RAT. In this arrangement, users will always be associated with the umbrella RAT, thus eliminating the need for a vertical emergency handover. In addition, when a conventional cell formation is constructed, providing control information from the umbrella RAT is capable of eliminating the horizontal handover, when users are moving across VLC cells/clusters. Nevertheless, when amorphous user-centric cells are constructed, horizontal handover become less frequent, since a moving cell is formed.

The above-mentioned data and control decoupling improves the mobility, while additionally contributing to the efficient resource management of VLC by forming user-specific AP combinations, by providing user-scheduling information, by adapting the physical layer parameters, etc. Further benefits arise when investigating the control signalling itself in detail, where sophisticated data and control decoupling takes into account the profiles of different traffic types, including their rate requirements, delay tolerance, relative inter-arrival frequencies, etc. [15]. More explicitly, as seen in Fig. 4, the control signalling of low data to control rate ratio (DCRR) traffic types, such as voice over IP (VoIP), is associated with the umbrella RAT, which provides a direct UL connection for facilitating lowlatency control signalling exchange. On the other hand, the control signalling of high DCRR traffic types, such as video streaming, can still be attached to the VLC link with the mobility-related control signalling being conveyed by the umbrella RAT.

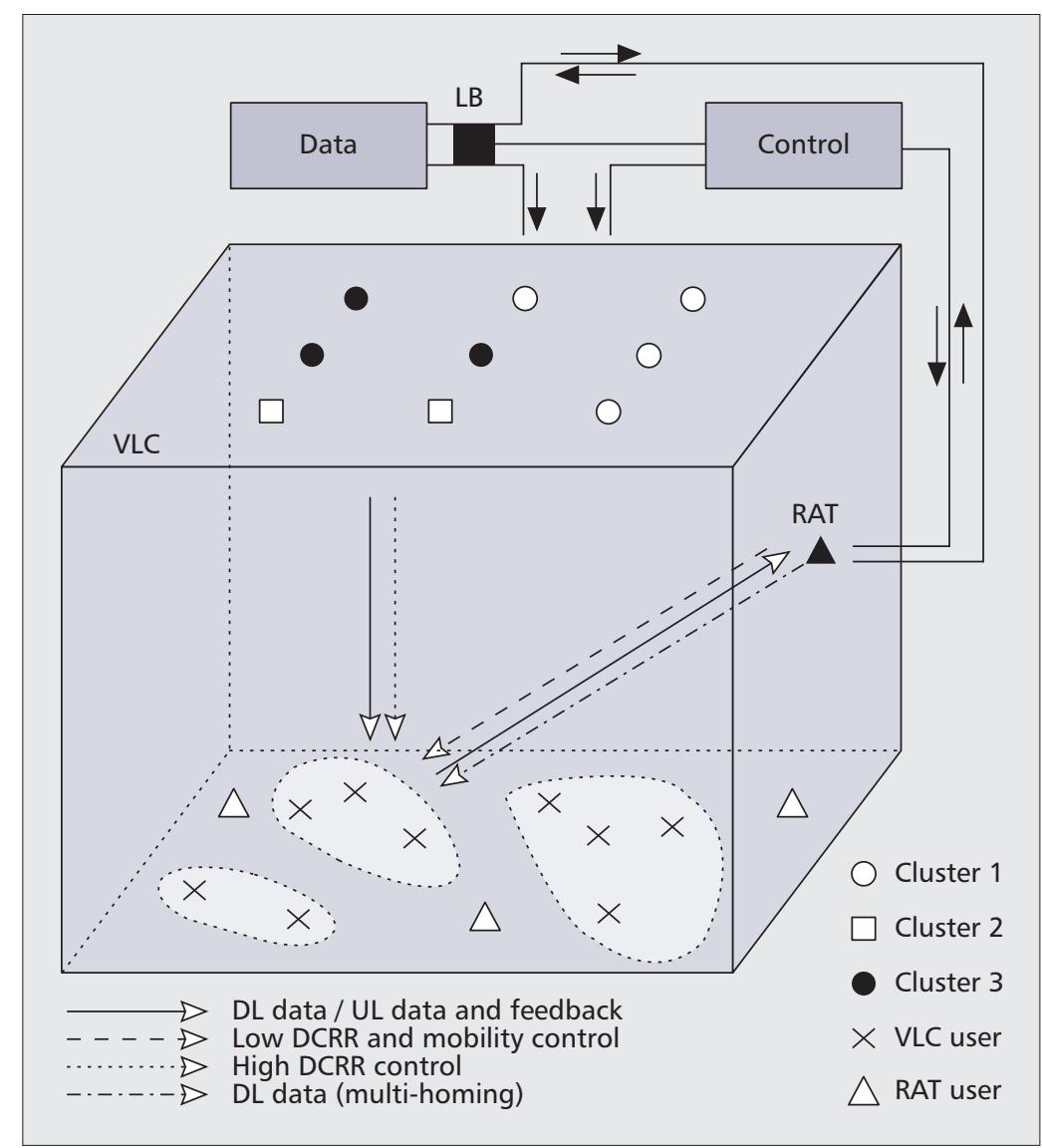

Figure 4. Indoor HetNet environment consisting VLC and RAT relying on a user-centric design, including amorphous cell formation, VLC DL and RAT UL, decoupled data and control as well as multi-homing capability.

\section{BaLANCED TRAFFIC LOAD}

When VLC is malfunctioning or its DL service is in outage, a fall-back RAT DL can be activated, with or without vertical handover, as discussed previously, which can be termed as a passive remedial approach. By contrast, the preventive proactive approach is to use dynamic load balancing (LB) between the VLC and the RAT DL access systems [12], as seen in Fig. 4. This arrangement will prevent traffic congestion in any of the two stand-alone access systems by diverting the traffic flow appropriately. However, the underlying mathematical problem is a complicated joint access system association and resource allocation optimization problem. More explicitly, one has to find the best association between the users and the pair of access systems, and then identify the optimal resource allocation for each association, in order to meet the various constraints, such as the fairness, rate, delay requirements, etc. To elaborate further, different users may require different services, including diverse data rates, and have various delay targets. From a system perspective, the proportional fairness among users is also an important design metric. Fortunately, there are efficient algorithms for solving this problem. For example, the original non-convex problem can be solved by proper transformation and dualdecomposition in order to arrive at a distributed solution. 


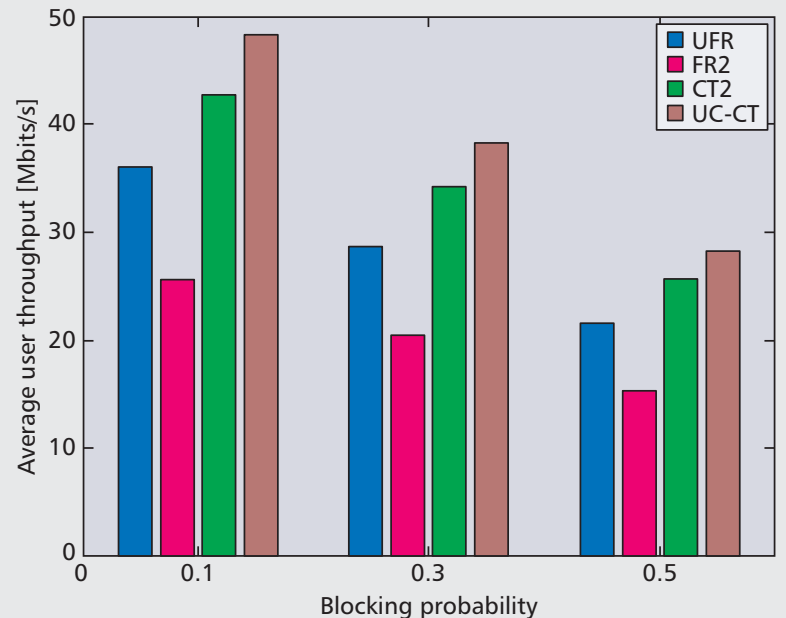

(a)
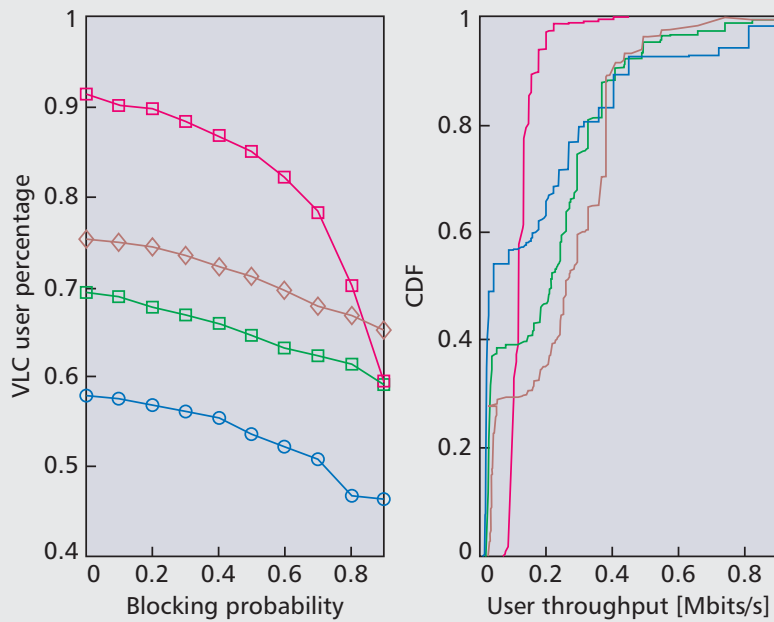

(b)

Figure 5. LB performance of a $3 \mathrm{~m} 15 \mathrm{~m} 15 \mathrm{~m}$ indoor heterogeneous VLC and WiFi 802.11 n environment having $4 \times 4$ uniformly distributed VLC APs with FoV of $120^{\circ}$. Four cell formations are investigated, namely the the UFR based as well as the FR 2 based regular design, the CT of merging two cells into one cluster and UC-CT: a) average user throughput of four different cell formations as a function of blocking probability; b) VLC user percentage of four different cell formations as a function of blocking probability (left) as well as the Cumulative Distribution Function (CDF) of user throughput of four different cell formations (right).

Figure 5 illustrates the LB performance of a $3 \mathrm{~m} \times 15 \mathrm{~m} \times 15 \mathrm{~m}$ indoor heterogeneous VLC and WiFi 802.11n environment having $4 \times 4$ uniformly distributed VLC APs with FoV of $120^{\circ}$. The LoS blocking is modelled by taking into account the blocking probability of the received signal. For example, having a blocking probability of 0.2 implies that 20 percent of the time the users will only receive the reflected signal component. It can be clearly seen in Fig. 5a that the average user throughout decreases upon increasing the LoS blocking probability for all four cell formations investigated, namely for the UFR based as well as for the FR2 based regular design, for the CT of merging two cells into one cluster and UC-CT. Moreover, the UC-CT based design exhibits the highest average user throughput for all LoS blocking probabilities investigated. To gain further insights, as seen at the left subplot of Fig. 5b, upon increasing the LoS blocking probability, fewer and fewer users are associated with VLC, while more and more users are shifted to WiFi. This scenario would result in increased outage in a stand-alone VLC network. More explicitly, cell formations having FR2 (denoted by the squares) retain the highest percentage of VLC users and are most affected by the LoS blocking. Such an arrangement leads to the lowest average user throughput, as seen in Fig. 5a, although it achieves the highest fairness, where most of the users have a throughput around the average value, as seen in the cumulative distribution function (CDF) in Fig. 5b when, for example, the LoS blocking probability is 0.1 . By contrast, the UFR based cell formation, the $\mathrm{CT}$ as well as UC-CT based cell formation exhibit a wide range of user throughputs, satisfying diverse user QoS requirements.

As far as the VLC and RAT access system association is concerned, we envision two different scenarios [13]: single-homing and multi-hom- ing. In a single-homing scenario, only one access system is allowed to maintain its association at any instant. To better exploit the access system's diversity potential in a multi-homing scenario, each user maintains multiple associations at the same time as seen in Fig. 4, where the underlying concept is reminiscent of the link aggregation philosophy of computer networks. Practically, the multi-homing operation can be supported with the aid of the Multipath Transport Control Protocol (TCP) under the recommendation RFC 6824 of the Internet Engineering Task Force (IETF), which is invoked both for maximizing the resource exploitation and for increasing the system's diversity gain by maintaining the TCP connection across multiple interfaces. This improves the robustness of the system, since encountering the event when both access systems are in outage is of low probability. Consequently, with the advent of multipath TCP, the outage of one of the access systems will not interrupt the end-toend TCP connection, hence leading to an improved QoS.

\section{Open Challenges}

In closing, let us finally elaborate on the open challenges of user-centric design.

\section{Native UL Design}

Although as discussed earlier, the classic RAT constitutes a viable solution for the UL of VLC aided HetNets, developing high-speed native optical wireless air-interfaces relying on infra-red or visible light for the UL is equally attractive. An optical transmitter may beam its UL information to a set of associated APs, different from those that serve the users in the DL. This leads to link asymmetry. For example, users can be equipped with a wide FoV receiver but narrow- 
beam transmitter or limited transmit power. A potential problem of the link asymmetry is the handover inconsistency, which is a direct result of the disparate UL and DL serving APs. The judicious design of optical wireless medium access control (MAC) protocols requires the optimization of cross-layer parameters and relies on a carefully decoupled data and control design from a user-centric perspective.

\section{Bi-DiRECTIONAL LOAD BALANCING}

In addition to dynamic LB for DL service provision, which was discussed earlier, coupling a VLC beam and a radio link in a joint optical wireless and RAT environment for the UL offers higher degrees of freedom and may lead to sophisticated network resource optimization. This design becomes more promising when the UL experiences heavy traffic due to mobile multimedia transmission in the near future, as a mobile unit becomes more and more powerful in terms of its computing power and storage. Thus, appropriately distributing heavy traffic in both the UL and DL requires efficient traffic reshaping, which is an unknown problem in existing mobile networks. Together with traffic imbalance among users, sophisticated bi-directional LB can be required in order to choose/aggregate the best available UL and DL access options.

\section{LOS BLOCKING}

LoS blocking is a severe problem in VLC systems, which is caused by the random movements of users and of obstructing objects. Immediately switching between access systems once blocking is encountered may not be the smartest approach, because it might result in potential ping-pong effects. Instead, a predictive decision should be adopted in order to mitigate the amount of control signalling and to reduce latency [18]. For example, when LoS blocking occurs, users engaged in delay tolerant services might prefer to maintain their VLC association if there is a high probability of having a recovering optical channel. By contrast, users engaged in real-time services would like to switch immediately from VLC to the umbrella RAT, if there is high probability of lasting VLC blocking. Therefore, the associated user-specific blocking patterns play an important role. More explicitly, to capture and predict the blocking patterns caused by the users' random movements, a Kalman filter can be used to track the movement of the user, and Markov chain based modelling can be invoked to predict the probability of LoS blocking, based on which an improved access system association and resource allocation decision can be achieved.

\section{SMART BACK-HAUL}

Another important design issue of VLC systems is that of the back-haul. There are numerous back-haul choices for VLC systems, but the most straightforward one relies on the power-line. However, due to the highly dispersive and noisy nature of the power-line, the attainable rate of the power-line limits that of the VLC system. On the other hand, fiber-based alternatives can be adopted as high-rate back-haul for VLC systems provided that its cost is affordable. Among others, a copper-based back-haul used for VLC sys- tems may constitute a promising solution. The most advanced copper technology relies on the G.fast standard (Recommendation ITU-T G.9701), which is capable of offering a Gigabits/sec throughput, thus it strikes a compelling balance between its cost and rate. As far as the usage of back-haul is concerned, by taking into account the geographically-disparate and timevarying tele-traffic demand of small-cells constituted by heterogeneous VLC APs and RAT, the precious system resources can be more efficiently exploited.

\section{CONCLUSIONS}

Incorporating VLC in HetNet environments constitutes a timely and promising enabler for resolving spectrum crunch and for improving the achievable network integrity. We advocated a user-centric design, with special emphasis on three key aspects, namely its signal coverage quality, system control, and service provision, which require a radically new perspective for the sake of improving the robustness of the system. More importantly, the user-centric design philosophy is also of high interest in both conventional RF based small-cells as well as in millimeter wave based small-cells. As a result, the user-centric design of VLC in HetNet environments constitutes a natural evolution.

\section{REFERENCES}

[1] T. Komine and M. Nakagawa, "Fundamental Analysis for Visible-Light Communication System Using LED Lights," IEEE Trans. Consumer Electronics, vol. 50, no. 1, Feb 2004, pp. 100-07.

[2] J. Grubor et al., "Broadband Information Broadcasting Using LED-based Interior Lighting," J. Lightwave Tech. vol. 26, no. 24, Dec 2008, pp. 3883-92.

[3] R. Zhang and L. Hanzo, "Multi-Layer Modulation for Intensity-Modulated Direct-Detection Optical OFDM," IEEE/OSA J. Opt. Commun. and Net., vol. 5, no. 12, Dec 2013, pp. 1402-12.

[4] Z. Wang et al., "An Adaptive Scaling and Biasing Scheme for OFDM-based Visible Light Communication Systems," Optics Express, vol. 22, no. 10, May 2014, pp. 12707-15.

[5] A. Azhar, T. Tran, and D. O'Brien, "A Gigabit/s Indoor Wireless Transmission Using MIMO-OFDM Visible-Light Communications," IEEE Photonics Tech. Lett., vol. 25 no. 2, Jan 2013, pp. 171-74.

[6] G. Cossu et al., "3.4 Gbit/s Visible Optical Wireless Transmission Based on RGB LED," Optics Express, vol. 20, no. 26, Dec 2012, pp. B501-B506.

[7] "Visible Light Communication (VLC)/LiFi Technology \& Free Space Optics (FSO) Markets (2013-2018)," Markets and Markets, Tech. Rep., Jan 2013.

[8] S. Hranilovic, L. Lampe, and S. Hosur, "Visible Light Communications: The Road to Standardization and Commercialization (Part 1)," IEEE Commun. Mag., vol. 51, no. 12, Dec 2013, pp. 24-25

[9] H. Burchardt et al., "VLC: Beyond Point-to-Point Communication," IEEE Commun. Mag., vol. 52, no. 7, July 2014, pp. 98-105.

[10] M. Rahaim, A. Vegni, and T. D. C. Little, "A Hybrid Radio Frequency and Broadcast Visible Light Communication system," IEEE GLOBECOM Wksps., Dec 2011, pp. 792-96.

[11] I. Stefan, H. Burchardt, and H. Haas, "Area Spectral Efficiency Performance Comparison between VLC and RF Femtocell Networks," IEEE Int'l. Conf. Commun., June 2013, pp. 3825-29.

[12] X. Li, R. Zhang, and L. Hanzo, "Cooperative Load Balancing in Hybrid Visible Light Communications and WiFi," IEEE Trans. Commun. (accepted), 2015. [Online]. Available: http://eprints.soton.ac.uk/365519/

[13] F. Jin, R. Zhang, and L. Hanzo, "Resource Allocation Under Delay-Guarantee Constraints for Heterogeneous Visible-Light and RF Femtocell," IEEE Trans. Wireless
More importantly, the user-centric design philosophy is also of high interests in both conventional RF based small-cells as well as in millimetre wave based small-cells. As a result, the user-centric design of VLC in HetNet environments constitutes a natural evolution. 
Commun. (accepted), 2015, available: http://eprints. soton.ac.uk/365518/.

[14] F. Boccardi et al., "Five Disruptive Technology Directions for 5G," IEEE Commun. Mag., vol. 52, no. 2, Feb 2014, pp. 74-80.

[15] C.-L. I et al., "Toward Green and Soft: A 5 G Perspective," IEEE Commun. Mag., vol. 52, no. 2, Feb. 2014 pp. 66-73.

[16] Y. Li et al., "VICO: A Framework for Configuring Indoor Visible Light Communication Networks," IEEE 9th Int'I. Conf. Mobile Adhoc and Sensor Systems (MASS), Oct 2012, pp. 136-44.

[17] X. Li et al., "Cell-Centric and User-Centric Multi-User Scheduling in Visible Light Communication Aided Networks," IEEE Int'l. Conf. Commun. (accepted), 2015, http://eprints soton.ac.uk/373461

[18] T. Nguyen, M. Z. Chowdhury, and Y. M. Jang, "A Novel Link Switching Scheme Using Pre-Scanning and RSS Prediction in Visible Light Communication Networks," EURASIP J. Wireless Communications and Networking, vol. 2013, no. 1, 2013, pp. 293-310.

\section{BIOGRAPHIES}

RONG ZHANG received his Ph.D. in 2009 from Southampton University, UK. He was a research assistant at Mobile Virtual Center of Excellence, UK, a research fellow at Southampton University, UK, and a system algorithms expert for Huawei Sweden R\&D. He is now a lecturer at Southampton Wireless Group of ECS, Southampton University. He has more than 40 published papers in prestigious publication avenues and many more in major conference proceedings. More details can be found at http://www.ecs.soton.ac.uk/ people/rz

JIAHENG WANG received the B.E. and M.S. degrees from Southeast University, Nanjing, China, in 2001 and 2006 respectively, and the Ph.D. degree in electrical engineering from The Hong Kong University of Science and Technology, Kowloon, Hong Kong, in 2010. He is currently an associate professor with the National Mobile Communications Research Laboratory (NCRL), Southeast University. From 2010 to 2011 he was with the Signal Processing Laboratory, ACCESS Linnaeus Center, KTH Royal Institute of Technology, Stockholm, Sweden. He also held a visiting position at the Department of Computer and Information Science, University of Macau, Macau. His research interests mainly include optimization in signal processing, wireless communications, and networks. He serves as an associate editor for IEEE Signal Processing Letters. He is a recipient of the Humboldt Fellowship for Experienced Researchers, and a recipient of the Best Paper Award in WCSP 2014.

ZHENGYUAN XU received his B.S. and M.S. degrees from Tsinghua University, China, and a Ph.D. degree from Stevens Institute of Technology, USA. He was with the University of California, Riverside, from 1999 to 2010, where he became a full professor with tenure and also a founding director of UC-Light Center. In 2010 he was selected by the Thousand Talents Program of China. He is professor and associate dean of the School of Information Science and Technology, University of Science and Technology of China. He is the founding director of the Wireless-Optical Communications Key Laboratory of Chinese Academy of Sciences, and chief scientist of the National Key Basic Research Program of China. His research focuses on wireless communication and networking, optical wireless communications, geolocation, and signal processing. He has published over 200 journal and conference papers. He was associate editor and guest editor for different IEEE journals, and founding co-chair of the IEEE GLOBECOM Workshop on Optical Wireless Communications. He has delivered tutorials, keynote speeches, and invited talks on optical wireless communications in various international conferences. He serves as associate editor for the OSA journal Photonics Research, and guest editor for IEEE JSAC special issue on Optical Wireless Communications.

ZHAOCHENG WANG received the B.S., M.S., and Ph.D. degrees from Tsinghua University, Beijing, China, in 1991, 1993 and 1996, respectively. From 1996 to 1997 he was a postdoctoral fellow with Nan yang Technological University, Singapore. From 1997 to 1999 he was with OKI Techno Centre Pte. Ltd., first as a research engineer and then as a senior engineer. From 1999 to 2009 he was with SONY Deutschland $\mathrm{GmbH}$, Germany, first as a senior engineer and then as a principal engineer. He is currently a professor with the Department of Electronic Engineering,
Tsinghua University and serves as director of the Broadband Communication Key Laboratory, Tsinghua National Laboratory for Information Science and Technology, P.R. China. His research interests include wireless communications, optical wireless communications, digital broadcasting, and millimeter wave communications. He is a holder of 33 granted U.S./EU patents and is the author of approximately $70 \mathrm{SCl}$ indexed journal papers. He is a Fellow of the Institution of Engineering and Technology (IET FELLOW) $\mathrm{He}$ is currently an editor of IEEE Transactions Wireless Communications, an editor of IEEE Communications Letters, and has served as technical program committee co-chair of many international conferences.

CHUNMING ZHAO received the B.S. and M.S. degrees from Nanjing Institute of Posts and Telecommunications, in 1982 and 1984, respectively. In 1993 he received the Ph.D. degree from the Department of Electrical and Electronic Engineering, University of Kaiserslautern, Germany. He has been a postdoctoral researcher at the National Mobile Communications Research Lab, Southeast University, where he is currently a professor and vice director of the Lab. His research interests include communication theory, coding/ decoding, mobile communications, and VLSI design. He has managed several key projects of the Chinese Communications High Tech. Program, and was received the "excellent researcher" award from the Ministry of Science and Technology, China. He also won the First Prize of National Technique Invention of China in 2011.

LAJOS HANZO is a Wolfson Fellow of the Royal Society, a Fellow of the Royal Academy of Engineering (FREng), FIEEE, FIET, and a EURASIP Fellow. He has co-authored 20 IEEE Press-John Wiley books, published more than 1400 research entries in IEEE Xplore, organized and chaired major IEEE conferences, and has been awarded a number of distinctions. More details can be found at http://wwwmobile.ecs.soton.ac.uk. 